\title{
Sudden Birth Versus Sudden Death of Entanglement in Multipartite Systems
}

\author{
C. E. López ${ }^{1}$, G. Romero ${ }^{1}$, F. Lastra ${ }^{2}$, E. Solano ${ }^{3,4}$ and J. C. Retamal ${ }^{1}$ \\ ${ }^{1}$ Departamento de Física, Universidad de Santiago de Chile, USACH, Casilla 307 Correo 2 Santiago, Chile \\ ${ }^{2}$ Facultad de Física, Pontificia Universidad Católica de Chile, Casilla 306, Santiago 22, Chile \\ ${ }^{3}$ Physics Department, ASC, and CeNS, Ludwig-Maximilians-Universität, Theresienstrasse 37, 80333 Munich, Germany \\ ${ }^{4}$ Departamento de Química Física, Universidad del País Vasco - Euskal Herriko Unibertsitatea, Apdo. 644, 48080 Bilbao, Spain
}

(Dated: October 31, 2018)

\begin{abstract}
We study the entanglement dynamics of two cavities interacting with independent reservoirs. Expectedly, as the cavity entanglement is depleted, it is transferred to the reservoir degrees of freedom. We find also that when the cavity entanglement suddenly disappear, the reservoir entanglement suddenly and necessarily appears. Surprisingly, we show that this entanglement sudden birth can manifest before, simultaneously, or even after entanglement sudden death. Finally, we present an explanatory study of other entanglement partitions and of higher dimensional systems.
\end{abstract}

PACS numbers: 03.65.Yz, 03.65.Ud, 03.67.Mn

Dynamical behavior of entanglement under the action of the environment is a central issue in quantum information [1, 2, 3, 4]. Recently, it has been observed that two qubits affected by uncorrelated reservoirs can experience disentanglement in a finite time despite coherence is lost asymptotically [2, 3, 4, 5, 6]. This phenomenon, called entanglement sudden death (ESD), has recently deserved a great attention $[7,8,19,10,11,12,13]$, and has been observed in the lab for entangled photon pairs [14], and atomic ensembles [15].

To our knowledge ESD has been studied mainly in relation to bipartite systems, while a deeper understanding is associated to the question of where does the lost entanglement finally go. This question would be properly answered by enlarging the system to include reservoir degrees of freedom. Intuitively, we may think that the lost entanglement has to be transferred to the reservoir degrees of freedom. However, is this entanglement swapped continuously? If the bipartite entanglement suffers ESD, what can we say about the transferred entanglement? Should there be a simultaneous entanglement sudden birth (ESB) on reservoir states, or when would this entanglement be created? In this work, we thoroughly study the entanglement transfer from the bipartite system to their independent reservoirs. We show that ESD of a bipartite system state is intimately linked to ESB of entanglement between the reservoirs, though their apparition times follow counterintuitive rules.

To illustrate the problem we have chosen the case of entangled cavity photons being affected by dissipation, as in the case of two modes inside the same dissipative cavity or single modes in two different ones. The present study could certainly be extended to other physical systems like matter qubits. First we study the case of qubits for two uncoupled (cavity) modes having up to one photon. Then, we extend our treatment to investigate wether or not the effect is present in higher dimensions (qudits).

Since each mode evolves independently, we can learn how to characterize the evolution of the overall system from the mode-reservoir dynamics. The interaction between a single cavity mode and an $N$-mode reservoir is described through the Hamiltonian

$$
\hat{H}=\hbar \omega \hat{a}^{\dagger} \hat{a}+\hbar \sum_{k=1}^{N} \omega_{k} \hat{b}^{\dagger} \hat{b}+\hbar \sum_{k=1}^{N} g_{k}\left(\hat{a} \hat{b}_{k}^{\dagger}+\hat{b}_{k} \hat{a}^{\dagger}\right) .
$$

Let us consider the case when a cavity mode is containing a single photon and its corresponding reservoir is in the vacuum state,

$$
\left|\phi_{0}\right\rangle=|1\rangle_{\mathrm{c}} \otimes|\overline{\mathbf{0}}\rangle_{\mathrm{r}}
$$

where, $|\overline{\mathbf{0}}\rangle_{\mathrm{r}}=\prod_{k=1}^{N}\left|0_{k}\right\rangle_{\mathrm{r}}$. It is not difficult to realize that the evolution given by (11) leads to the state

$$
\left|\phi_{t}\right\rangle_{\mathrm{cr}}=\xi(t)|1\rangle_{\mathrm{c}}|\overline{\mathbf{0}}\rangle_{\mathrm{r}}+\sum_{k=1}^{N} \lambda_{k}(t)|0\rangle_{\mathrm{c}}\left|1_{k}\right\rangle_{\mathrm{r}},
$$

where the state $\left|1_{k}\right\rangle_{\mathrm{r}}$ accounts for the reservoir having one photon in mode $k$. The amplitude $\xi(t)$ converges to $\xi(t)=\exp (-\kappa t / 2)$ in the limit of $N \rightarrow \infty$ for a reservoir with a flat spectrum. The right-hand term of the last equation can be rewritten in terms of a collective state of the reservoir modes as

$$
\left|\phi_{t}\right\rangle=\xi(t)|1\rangle_{\mathrm{c}}|\overline{\mathbf{0}}\rangle_{\mathrm{r}}+\chi(t)|0\rangle_{\mathrm{c}}|\overline{\mathbf{1}}\rangle_{\mathrm{r}}
$$

Here, we defined the normalized collective state with one excitation in the reservoir as

$$
|\overline{\mathbf{1}}\rangle_{\mathrm{r}}=\frac{1}{\chi(t)} \sum_{k=1}^{N} \lambda_{k}(t)\left|1_{k}\right\rangle_{\mathrm{r}},
$$

and the amplitude $\chi(t)$ in Eq. (44) converge to the expression $\chi(t)=(1-\exp (-\kappa t))^{1 / 2}$ in the large $N$ limit. Described in this way the cavity and reservoir evolve as an effective two-qubit system [16].

Let us now study the joint evolution of two qubits with their corresponding reservoirs initially in the global state

$$
\left|\Phi_{0}\right\rangle=\left(\alpha|0\rangle_{\mathrm{c}_{1}}|0\rangle_{\mathrm{c}_{2}}+\beta|1\rangle_{\mathrm{c}_{1}}|1\rangle_{\mathrm{c}_{2}}\right)|\overline{\mathbf{0}}\rangle_{\mathrm{r}_{1}}|\overline{\mathbf{0}}\rangle_{\mathrm{r}_{2}} .
$$


According to Eq.(4), the evolution of the overall system will be given by

$$
\begin{aligned}
\left|\Phi_{t}\right\rangle= & \alpha|0\rangle_{\mathrm{c}_{1}}|\overline{\mathbf{0}}\rangle_{\mathrm{r}_{1}}|0\rangle_{\mathrm{c}_{2}}|\overline{\mathbf{0}}\rangle_{\mathrm{r}_{2}} \\
& +\beta\left|\phi_{t}\right\rangle_{\mathrm{c}_{1} \mathrm{r}_{1}}\left|\phi_{t}\right\rangle_{\mathrm{c}_{2} \mathrm{r}_{2}} .
\end{aligned}
$$

We observe that the overall state evolves as a four-qubit system. By tracing out the reservoir states, the reduced two-cavity reduced density matrix reads

$$
\rho_{\mathrm{c}_{1} \mathrm{c}_{2}}=\left(\begin{array}{cccc}
\alpha^{2}+\beta^{2} \chi^{4} & 0 & 0 & \alpha \beta \xi^{2} \\
0 & \beta^{2} \xi^{2} \chi^{2} & 0 & 0 \\
0 & 0 & \beta^{2} \xi^{2} \chi^{2} & 0 \\
\alpha \beta \xi^{2} & 0 & 0 & \beta^{2} \xi^{4}
\end{array}\right) .
$$

This reduced state $\rho_{\mathrm{c}_{1} \mathrm{c}_{2}}$ has the structure of an $X$ matrix and exhibits ESD for $\alpha<\beta$ [5, 6]. On the other hand, when tracing out cavity modes we are led to the reduced reservoir state

$$
\rho_{\mathrm{r}_{1} \mathrm{r}_{2}}=\left(\begin{array}{cccc}
\alpha^{2}+\beta^{2} \xi^{4} & 0 & 0 & \alpha \beta \chi^{2} \\
0 & \beta^{2} \chi^{2} \xi^{2} & 0 & 0 \\
0 & 0 & \beta^{2} \xi^{2} \chi^{2} & 0 \\
\alpha \beta \chi^{2} & 0 & 0 & \beta^{2} \chi^{4}
\end{array}\right),
$$

whose structure also corresponds to an $X$ state. When replacing $\xi(t) \leftrightarrow \chi(t)$, this state is complementary to the state in Eq. (8). If $\rho_{\mathrm{c}_{1} \mathrm{c}_{2}}$ is exhibiting ESD, what happens then with $\rho_{\mathrm{r}_{1}{ }_{2}}$ ? To answer this question we calculate the concurrence [17] for $\rho_{\mathrm{c}_{1} \mathrm{c}_{2}}$, which for the particular state is given by the simple expression

$$
\mathcal{C}(t)=\max \{0,-2 \lambda\}
$$

with $\lambda$ being the negative eigenvalue of the density matrix partial transpose. For reduced states $\rho_{\mathrm{c}_{1} \mathrm{c}_{2}}$ and $\rho_{\mathrm{r}_{1} \mathrm{r}_{2}}$ these negative eigenvalues are given by

$$
\begin{aligned}
& \lambda_{\mathrm{c}_{1} \mathrm{c}_{2}}=e^{-\kappa t}\left[\beta^{2}\left(1-e^{-\kappa t}\right)-|\alpha \beta|\right], \\
& \lambda_{\mathrm{r}_{1} \mathrm{r}_{2}}=\left(1-e^{-\kappa t}\right)\left[\beta^{2} e^{-\kappa t}-|\alpha \beta|\right] .
\end{aligned}
$$

Figure 1 shows the evolution of concurrence between the two cavities (solid line) and the two reservoirs (dashed line). Despite the entanglement between the two cavities suddenly disappears, sudden birth of entanglement arises between the two reservoirs. Note that the entanglement contained initially in the cavity-cavity subsystem is transferred to the bipartite reservoir system. The time for which ESD and the entanglement sudden birth (ESB) occur can be calculated from Eqs. (11) and (12), looking for the time where $\lambda_{\mathrm{c}_{1} \mathrm{c}_{2}}$ becomes positive for ESD and the time for which $\lambda_{\mathrm{r}_{1} \mathrm{r}_{2}}$ becomes negative for ESB,

$$
\begin{aligned}
& t_{\mathrm{ESD}}=-\frac{1}{\kappa} \ln \left(1-\frac{\alpha}{\beta}\right), \\
& t_{\mathrm{ESB}}=\frac{1}{\kappa} \ln \frac{\beta}{\alpha} .
\end{aligned}
$$

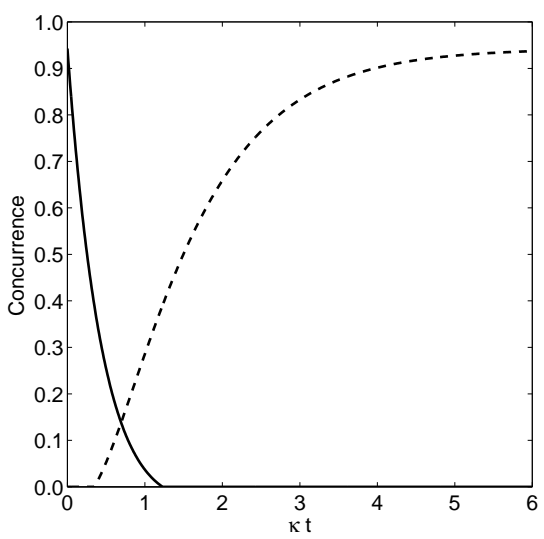

FIG. 1: Evolution of two-qubit concurrence $\mathcal{C}_{\mathrm{c}_{1} \mathrm{c}_{2}}$ (solid line) and $\mathcal{C}_{\mathrm{r}_{1} \mathrm{r}_{2}}$ (dashed line), for the initial state of Eq. (66) with $\alpha=1 / \sqrt{3}$ and $\beta=2 / \sqrt{3}$.

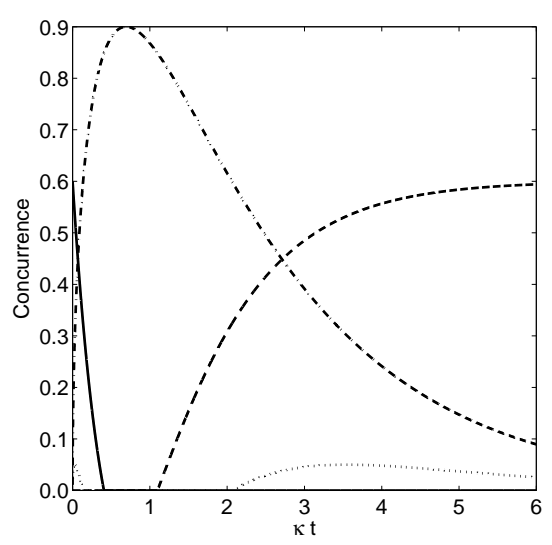

FIG. 2: Evolution of two-qubit concurrence for different partitions: $\mathcal{C}_{\mathrm{c}_{1} \mathrm{c}_{2}}$ (solid line), $\mathcal{C}_{\mathrm{r}_{1} \mathrm{r}_{2}}$ (dashed line), $\mathcal{C}_{\mathrm{c}_{1} \mathrm{r}_{1}}$ (dot-dashed line), $\mathcal{C}_{\mathrm{c}_{1} \mathrm{r}_{2}}$ (dotted line), for the initial state of Eq. (6) with $\alpha=1 / \sqrt{10}$ and $\beta=3 / \sqrt{10}$.

From these expressions we learn that ESB occurs for $\beta>\alpha$, as is the case for ESD. In other words, the presence of ESD implies necessarily the apparition of ESB and, consequently, asymptotic decay of entanglement between cavities implies an asymptotic birth and growing of entanglement between reservoirs.

For the situation in Fig. 1 we have $t_{\mathrm{ESB}}<t_{\mathrm{ESD}}$. However, as can be easily seen from Eqs. (13), when $\beta=2 \alpha$, $t_{\mathrm{ESB}}=t_{\mathrm{ESD}}$, that is, ESB and ESD happen simultaneously. Furthermore, when $\beta>2 \alpha$, ESB occurs after ESD. Although this is clear from Eqs. (13), it is not necessarily intuitive. In fact, this condition yields a time window where neither the cavity-cavity nor the reservoirreservoir subsystems have entanglement.

To have an idea of how the entanglement is shared among the parties, we study the entanglement present in different partitions. We start considering all bipartite partitions of two qubits, namely: $c_{1} \otimes c_{2}, r_{1} \otimes r_{2}, c_{1} \otimes r_{1}$ and $c_{1} \otimes r_{2}$, as shown in Fig. 2. In particular for partition 


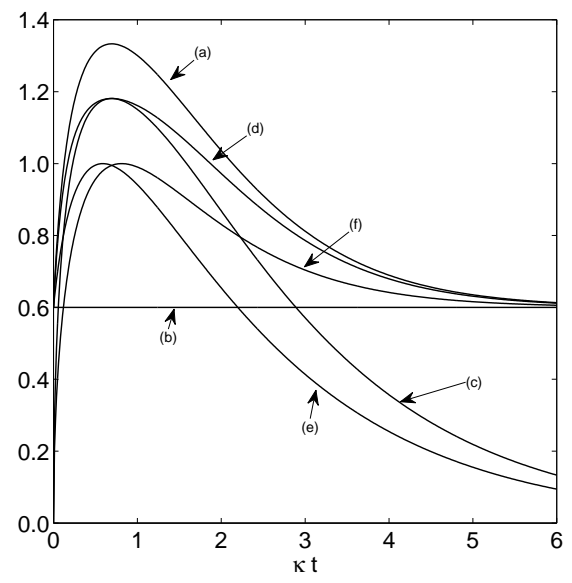

FIG. 3: Evolution of entanglement for different partitions: (a) $c_{1} \otimes r_{1} \otimes c_{2} \otimes r_{2} ;(\mathrm{b})\left(c_{1} \otimes r_{1}\right) \otimes\left(c_{2} \otimes r_{2}\right) ;(\mathrm{c})\left(c_{1} \otimes c_{2}\right) \otimes\left(r_{1} \otimes r_{2}\right) ;$ (d) $\left(c_{1} \otimes r_{2}\right) \otimes\left(c_{2} \otimes r_{1}\right) ;(\mathrm{e}) c_{1} \otimes\left(r_{1} \otimes c_{2} \otimes r_{2}\right) ;(\mathrm{f}) r_{1} \otimes\left(c_{1} \otimes c_{2} \otimes r_{2}\right)$ for the initial state of Eq. (6) with $\alpha=1 / \sqrt{10}$ and $\beta=3 / \sqrt{10}$.

$c_{1} \otimes r_{1}$, the entanglement is given by

$$
\mathcal{C}_{\mathrm{c}_{1} \mathrm{r}_{1}}(t)=2 \beta^{2} \sqrt{\left(1-e^{-\kappa t}\right) e^{-\kappa t}} .
$$

In the region where there is no entanglement, that is, $\mathcal{C}_{\mathrm{c}_{1} \mathrm{c}_{2}}=\mathcal{C}_{\mathrm{r}_{1} \mathrm{r}_{2}}=0$, entanglement between a cavity and its corresponding reservoir $\mathcal{C}_{\mathrm{c}_{1} \mathrm{r}_{1}}(t)$ reaches its maximum value. This fact is independent of the initial probability amplitudes $\alpha$ and $\beta$ and occurs for a time $t=\kappa^{-1} \ln (2)$ which corresponds also to the time when $t_{\mathrm{ESD}}=t_{\mathrm{ESB}}$.

Entanglement of other bipartite partitions is shown in Fig. 3(b)-(f), and the multipartite entanglement between the four effective qubits in Fig. 31(a). Such entanglement is described by the multipartite concurrence $\mathcal{C}_{N}$ [18]. For partitions (b)-(f) the entanglement is obtained through the square root of the tangle [19] which in the pure twoqubit case coincides with the concurrence. Note that $\mathcal{C}_{N}$ has the same value at $t=0$ and $t \rightarrow \infty$, showing complete entanglement transfer from cavities to reservoirs.

Although entanglement transfer from cavities to reservoirs is mediated only by the interaction of each cavity and its corresponding reservoir, entanglement may also flow through other parties. Figure 3(b) shows that the partition $\left(c_{1} \otimes r_{1}\right) \otimes\left(c_{2} \otimes r_{2}\right)$ has constant entanglement. However, Fig. 2 shows that entanglement in the two-qubit partition $c_{1} \otimes r_{2}$ is created along the evolution, implying that entanglement flows also to the noninteracting partitions. This fact can be visualized as follows: initially the entanglement is contained in the partition $c_{1} \otimes c_{2}$. Then, due to the interaction between cavities and reservoirs, for example $c_{1}$ and $r_{1}$, the information about the quantum state of $c_{1}$ begins to be mapped into the quantum state of $r_{1}$. Therefore, some of the quantum information contained in the joint-system of the cavities [5] is now present in the joint-system of $c_{2} \otimes r_{1}$, producing entanglement in this partition.
It is interesting to investigate whether the features we have analyzed so far are present for higher dimensional systems. For example, we consider the case of qutrit cavity states. Following similar steps used to obtain Eq. (4), it is not difficult to calculate the evolution of a single cavity mode, initially in a two-photon $|2\rangle_{\mathrm{c}}$ state, interacting with the reservoir initially in the vacuum state. The initial state $\left|\phi_{0}^{(2)}\right\rangle=|2\rangle \otimes|\overline{\mathbf{0}}\rangle$ evolves according with $\left|\phi_{t}^{(2)}\right\rangle=\xi^{2}(t)|2\rangle_{\mathrm{c}}|\overline{\mathbf{0}}\rangle_{\mathrm{r}}+\sqrt{2} \xi(t) \chi(t)|1\rangle_{\mathrm{c}}|\overline{\mathbf{1}}\rangle_{\mathrm{r}}+\vartheta(t)|0\rangle_{\mathrm{c}}|\overline{\mathbf{2}}\rangle_{\mathrm{r}}$,

where,

$$
\begin{aligned}
|\overline{\mathbf{2}}\rangle_{\mathrm{r}}= & \frac{1}{\vartheta(t)}\left(\sum_{k=1}^{N}\left|\lambda_{k}(t)\right|^{2}\left|2_{k}\right\rangle\right. \\
& \left.+\sqrt{2} \sum_{k \neq q=1}^{N} \lambda_{k}(t) \lambda_{q}(t)\left|1_{k} \ldots 1_{q}\right\rangle_{\mathrm{r}}\right),
\end{aligned}
$$

and $\vartheta(t)=\sqrt{1-\xi^{4}(t)-2 \xi^{2}(t) \chi^{2}(t)}$. We can now study the entanglement when the initial state is given by

$$
\left|\Phi_{0}\right\rangle=\left(\alpha|0\rangle_{\mathrm{c}_{1}}|0\rangle_{\mathrm{c}_{2}}+\beta|1\rangle_{\mathrm{c}_{1}}|1\rangle_{\mathrm{c}_{2}}+\gamma|2\rangle_{\mathrm{c}_{1}}|2\rangle_{\mathrm{c}_{2}}\right) \otimes|\overline{\mathbf{0}}\rangle_{\mathrm{r}_{1}}|\overline{\mathbf{0}}\rangle_{\mathrm{r}_{2}} .
$$

As no entanglement monotone exists for an arbitrary higher dimensional state, we focus on the analytical expression for a lower bound of entanglement (LBOE) found by Chen, et. al. 20], based on the PPT [21, 22] and realignment criterion [23, 24]. The LBOE monotone of a bipartite system (A and B) denoted $\Lambda$ is given by $\Lambda=\max \left(\left\|\rho^{T_{A}}\right\|,\|R(\rho)\|\right)$, where the trace norm $\|\cdot\|$ is defined as $\|G\|=\operatorname{tr}\left(G G^{\dagger}\right)^{\frac{1}{2}}$. The matrix $\rho^{T_{A}}$ is the partial transpose with respect to the subsystem $A$, that is, $\rho_{i k, j l}^{T_{A}}=\rho_{j k, i l}$, and the matrix $R(\rho)$ is realignment matrix defined as $R(\rho)_{i j, k l}=\rho_{i k, j l}$. The values of $\Lambda$ ranges from 1 (separable state) to $d$ (maximally entangled), where $d$ is the dimension of the lower dimensional subsystem.

In Fig. (4), the evolution of $\Lambda_{\mathrm{c}_{1} \mathrm{c}_{2}}(t)$ and $\Lambda_{\mathrm{r}_{1} \mathrm{r}_{2}}(t)$ is shown. We observe that the sudden death of the cavitycavity entanglement is accompanied by sudden birth of reservoir-reservoir entanglement as in the two-qubit case. Moreover, the LBOE dynamics between the reservoirs exhibits abrupt changes as the LBOE between cavities [13]. The times for the ESD and the ESB to appear are

$$
\begin{aligned}
& t_{\mathrm{ESD}}^{\mathrm{c}_{1} \mathrm{c}_{2}}=-\frac{1}{\kappa} \ln \left(1-\left(\frac{\alpha}{\gamma}\right)^{\frac{1}{2}}\right), \\
& t_{\mathrm{ESB}}^{\mathrm{r}_{1} \mathrm{r}_{2}}=\frac{1}{2 \kappa} \ln \frac{\gamma}{\alpha} .
\end{aligned}
$$

As for the two-qubit case, the time for wich ESD and ESB occur simultaneously results to be $t=\kappa^{-1} \ln (2)$.

In general, for a $d \otimes d$-dimensional bipartite system, each one coupled to an independent reservoir, and initially prepared in a state of the form

$$
\left|\Psi_{0}\right\rangle=\sum_{k=0}^{d} \alpha_{k}|k\rangle_{\mathrm{c}_{1}}|k\rangle_{\mathrm{c}_{2}} \otimes|\overline{\mathbf{0}}\rangle_{\mathrm{r}_{1}}|\overline{\mathbf{0}}\rangle_{\mathrm{r}_{2}}
$$




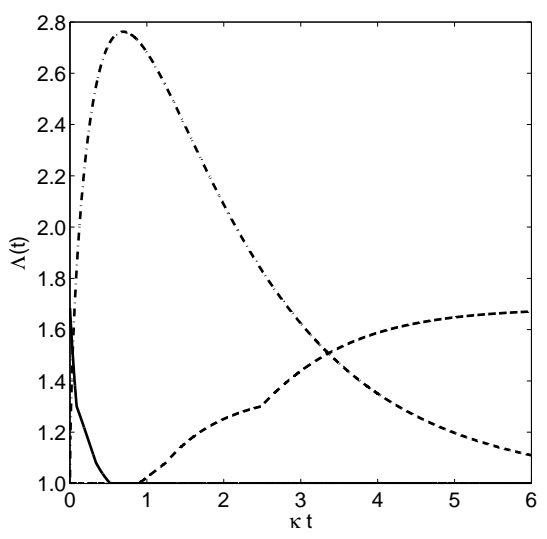

FIG. 4: Evolution of two-qutrit LBOE for different partitions: $\Lambda_{\mathrm{c}_{1} \mathrm{c}_{2}}$ (solid line), $\Lambda_{\mathrm{r}_{1} \mathrm{r}_{2}}$ (dashed line), $\Lambda_{\mathrm{c}_{1} \mathrm{r}_{1}}$ (dot-dashed line), $\Lambda_{\mathrm{c}_{1} \mathrm{r}_{2}}$ (dotted line), for the initial state of Eq. (17) with $\alpha=$ $1 / \sqrt{38}$, and $\beta=1 / \sqrt{38}$ and $\gamma=6 / \sqrt{38}$.

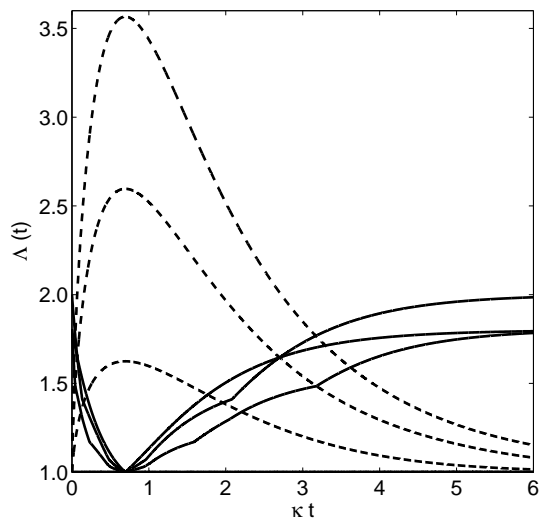

FIG. 5: Evolution of the LBOE $\Lambda_{\mathrm{c}_{1} \mathrm{c}_{2}}$ and $\Lambda_{\mathrm{r}_{1} \mathrm{r}_{2}}$ (solid lines) and $\Lambda_{\mathrm{c}_{1} \mathrm{r}_{1}}$ (dashed lines) for initial state of Eq. (20) with $d=$ 2,3 , and 4 . Probability amplitudes $\alpha_{k}$ with $k=0,1, . ., d-1$ are all equals to $1 / \sqrt{\sum_{k=0}^{d}\left|\alpha_{k}\right|^{2}}$ and $\alpha_{d}=2^{d-1} \alpha_{0}$.

we have numerically observed that the time when $t_{\mathrm{ESD}}=$ $t_{\mathrm{ESB}}$ does not depend on the dimension of the systems. As can be seen from Fig. 5 the time for which $t_{\mathrm{ESD}}=t_{\mathrm{ESD}}=\kappa^{-1} \ln (2)$. The necessary condition for these times to be equal is $\alpha_{d} / \alpha_{0}=2^{d-1}$. Although this condition does not depend on the remaining probability amplitudes $\alpha_{k}$ with $k \neq 0, d$, the condition $\alpha_{k}<\alpha_{d}$ must be satisfied to ensure the presence of ESD and ESB.

In conclusion, we have shown that ESD in a bipartite system independently coupled to two reservoirs is necessarily related to the ESB between the environments. The loss of entanglement is related to the birth of entanglement between the reservoirs and other partitions. We analytically demonstrate that ESD and ESB occur at times depending on the amplitudes of the initial entangled state. We found that ESB occur before, together, or even after ESD. In the latter case, when neither cavities nor reservoirs have entanglement, we have analyzed how the entanglement flows to other partitions. Finally, we showed that the simultaneous occurrence of ESD and ESB is independent of the system dimension.

C.E.L. acknowledges financial support from Fondecyt 11070244, DICYT USACH and PBCT-CONICYT PSD54, F.L. from Fondecyt 3085030, G.R. from CONICYT grants, J.C.R. from Fondecyt 1070157 and Milenio ICM P06-067, E.S. from SFB 631, EU EuroSQIP, and Ikerbasque Foundation.

[1] T. S. Cubitt, F. Verstraete, W. Dür, and J. I. Cirac , Phys. Rev. Lett. 91, 037902 (2003); T. S. Cubitt, F. Verstraete, and J. I. Cirac , Phys. Rev. A 71, 052308 (2005).

[2] L. Diósi, Lect. Notes Phys. 622, 157-163 (2003).

[3] P. J. Dodd and J. J. Halliwell, Phys. Rev. A, 69, 052105 (2004).

[4] A. R. R. Carvalho, F. Mintert, S. Palzer, and A. Buchleitner, Eur. Phys. J. D 41, 425-432 (2007).

[5] Ting Yu and J. H. Eberly, Phys. Rev. Lett. 93, 140404 (2004); ibid. 97, 140403 (2006).

[6] M.F. Santos, P. Milman, L. Davidovich, and N. Zagury, Phys. Rev. A. 73, 040305(R), 2006.

[7] Anna Jamróz, J. Phys. A 39, 7727 (2006).

[8] Z. Ficek and R. Tanaś, Phys. Rev. A 74, 024304 (2006).

[9] M. O. Terra Cunha, New J. Phys. 9, 237 (2007).

[10] A. Al-Qasimi and D. F. V. James, Phys. Rev. A 77, 012117 (2008); Ting Yu and J. H. Eberly, arXiv:quantph/0707.3215; L. Roa, R. Pozo-González, M. Schaefer, and P. Utreras-SM, Phys. Rev. A 75, 062316 (2007); H. T. Cui, K. Li, and X. X. Yi, arXiv:quant-ph/0612145 F. F. Fanchini and R. d. J. Napolitano, arXiv:quantph/0707.4092; A. R. P. Rau, M. Ali, and G. Alber, arXiv:quant-ph/0711.0317.

[11] L. Derkacz and L. Jakóbczyk, Phys. Rev. A 74, 032313 (2006).

[12] Z. Sun, X. Wang, and C. P. Sun, Phys. Rev. A 75, 062312 (2007).

[13] F. Lastra, G. Romero, C. E. López, M. França Santos, and J. C. Retamal, Phys. Rev. A 75, 062324 (2007).

[14] M. P. Almeida, et al., Sience 316, 579 (2007).

[15] J. Laurat, K.S Choi, H. Deng, C.W. Chou, H.J. Kimble, Phys. Rev. Lett. 99, 180504 (2007).

[16] Note that this approach is different from the case of four qubits in the purely unitary case. See M. Yönaç, Ting Yu and J. H. Eberly, J. Phys. B 40, S45 (2007)

[17] W. K. Wootters, Phys. Rev. Lett. 80, 2245 (1998).

[18] A.R.R Carvalho, F. Mintert, and A. Buchleitner, Phys. Rev. Lett. 93, 230501 (2004).

[19] P. Rungta, V. Buzek, C.M. Caves, M. Hillery, and G.J. Milburn, Phys. Rev. A 64, 042315 (2003).

[20] K. Chen, S. Albeverio, S.M. Fei, Phys. Rev. Lett. 95, 210501 (2005).

[21] A. Peres, Phys. Rev. Lett. 77, 1413 (1996).

[22] M. Horodecki, P. Horodecki, R. Horodecki, Phys. Lett. A 223, 1 (1996).

[23] O. Rudolph, arXiv:quant-ph/0202121.

[24] K. Chen, L. A. Wu, Quantum Inf. Comput. 3, 193 (1999). 